\title{
The ultrametric space of plane branches
}

\author{
Ignasi Abío, Maria Alberich-Carramiñana and Víctor González-Alonso \\ Departament de Matemàtica Aplicada I, Universitat Politècnica de Catalunya, \\ Av. Diagonal 647, 08028-Barcelona, Spain. \\ Maria Alberich-Carramiñana is also with the \\ Institut de Robòtica i Informàtica Industrial (CSIC-UPC), \\ Llorens i Artigues 4-6, 08028 Barcelona, Spain \\ e-mail: ignasi.abio@upc.edu, maria.alberich@upc.edu, victor.gonzalez-alonso@upc.edu *
}

\begin{abstract}
We study properties of the space of irreducible germs of plane curves (branches), seen as an ultrametric space. We provide various geometrical methods to measure the distance between two branches and to compare distances between branches, in terms of topological invariants of the singularity which comprises some of the branches. We show that, in spite of being very close to the notion of intersection multiplicity between two germs, this notion of distance behaves very differently. For instance, any value in $[0,1] \cap \mathbb{Q}$ is attained as the distance between a fixed branch and some other branch, in contrast with the fact that the semigroup of the fixed branch has gaps. We also present results that lead to interpret this distance as a sort of geometric distance between the topological equivalence or equisingularity classes of branches.
\end{abstract}

\section{Introduction}

The notion of local intersection multiplicity between two analytic germs of curve $C: f=0$ and $D: g=0$ defined in a neighbourhood of the origin $O$ of $\mathbb{C}^{2}$,

$$
[C \cdot D]_{O}=\operatorname{dim}_{\mathbb{C}} \frac{\mathbb{C}\{x, y\}}{(f, g)},
$$

admits a geometric interpretation after Noether's intersection formula (see the revised approach of [1] 3.3.1), which exhibits that $[C \cdot D]_{O}$ accounts for the

*This research has been partially supported by the Spanish Committee for Science and Technology (CAICYT) through the project M2009-14163-C02-02, and the Catalan Research Commission through the project 2009 SGR 1284. Ignasi Abío and Víctor González-Alonso completed this work supported each of them by a postgraduate scholarship of the FPU program from the Spanish Ministerio de Educación y Ciencia. 
infinitely near points shared by both curves, namely:

$$
[C \cdot D]_{O}=\sum_{p \in \mathcal{N}(C) \cap \mathcal{N}(D)} m_{p}(C) m_{p}(D)
$$

the summation running on the infinitely near points $p$ lying both on $C$ (denoted by $\mathcal{N}(C)$ ) and on $D, m_{p}(C)$ denoting the multiplicity at $p$ of the strict transform of the curve $C$ on a surface where $p$ is a proper point.

Consider the set $\mathcal{C}$ of irreducible analytic germs of curve in $\left(\mathbb{C}^{2}, O\right)$, which will also be called branches. We remark that all the notions and results of this paper apply also in the case of formal germs of curves by simply substituting the analytic ring $\mathbb{C}\{x, y\}$ by the formal ring $\mathbb{C} \llbracket x, y \rrbracket$. Hence, once a pair of local coordinates $x, y$ in $\left(\mathbb{C}^{2}, O\right)$ is chosen, a branch is defined by a class of equations determined by $f \in \mathbb{C}\{x, y\}$ modulo an invertible. We will refer to $\mathcal{C}$ as the space of plane branches, since it will be endowed with a topology defined by a distance, as it will be showed next. Set $n_{p}(C)=\frac{m_{p}(C)}{m_{O}(C)}$, the normalized multiplicity of $C$ at the infinitely near point $p$. When applying Noether's intersection formula to a pair of distinct branches $C, D \in \mathcal{C}$ by considering normalized multiplicities instead of multiplicities, we obtain

$$
\frac{[C \cdot D]_{O}}{m_{O}(C) m_{O}(D)}=\sum_{p \in \mathcal{N}(C) \cap \mathcal{N}(D)} n_{p}(C) n_{p}(D)=\frac{1}{d_{\mathcal{C}}(C, D)}
$$

and its inverse $d_{\mathcal{C}}(C, D)$ is an ultrametric distance in the set of plane branches $\mathcal{C}$ (of course, we set $d_{\mathcal{C}}(C, C)=0$; see [5]). This notion is very close to the notion of contact between two germs, which plays a fundamental role in the theory of polar germs of plane curves (see [6] 2.4 or [2] 5.2 or [7] pg. 69). More precisely, the geometrical methods to measure distances presented in this paper have revealed to be key tools to solve deep problems on polars, which will be the object of a forthcoming paper.

Having the definition of $d_{\mathcal{C}}$ (namely, equation (1)) in mind, one might feel tempted to conclude that the notion $d_{\mathcal{C}}$ should not be very distant from that of taking inverse of the intersection multiplicity, and hence one might hope that, given a branch $C$, the set

$$
\Delta_{C}:=\left\{d \in[0,1] \cap \mathbb{Q}: d=d_{C}(C, D), D \in \mathcal{C}\right\}
$$

should be like a sort of inverse of the positive values of the semigroup $\Sigma_{C}$ of the branch $C$. But this is far from being true: we prove here that $\Delta_{C}=$ $[0,1] \cap \mathbb{Q}$, whereas it is widely known that $\Sigma_{C}$ has gaps (values not attained as intersection multiplicity of $C$ and any germ) closely related to the singularity of $C$ (see [1] 5.8.7). Moreover, on one side $\Sigma_{C}$ is an equisingularity (or topological) invariant which determines in turn the equisingularity class of $C$; on the other side, a given value $a \in \Sigma_{C}$ may be attained by various branches having nonconnected equisingularity classes (see [1] §5.8). In contrast, we show in Theorem 2.7 and Corollary 2.8 that any value $d \in \Delta_{C}$ attained by a branch $D$ forces the equisingularity class of $D$ to be so "close" to that of $C$ as the value $d$ determines, 
where "close" has a geometric meaning: the Enriques diagram (which is a rooted graph encoding the topological equivalence or equisingularity class of a plane curve singularity) of both branches share the more vertices, the smaller $d$ is. This fact could be interpreted as saying that $d_{C}$ plays the role of a geometric distance between the topological equivalence or equisingularity classes of branches.

The distance $d_{\mathcal{C}}$ is also closely related to the notion of skewness of a valuation of $\mathbb{C}\{x, y\}$ or $\mathbb{C} \llbracket x, y \rrbracket$ appearing in the recent development of the valuative theory of [4]. Namely, the valuation $\nu_{C}$ attached to a branch $C$ is defined as $\nu_{C}(\psi)=$ $\frac{[C \cdot(\psi=0)]_{O}}{m_{O}(C)}$; the valuation $\nu_{C} \wedge \nu_{D}$ attached to a pair of branches $C$ and $D$ is defined as $\left(\nu_{C} \wedge \nu_{D}\right)(\psi)=\min \left\{\nu_{C}(\psi), \nu_{D}(\psi)\right\}$ for any $\psi \in(x, y)$ irreducible; the skewness of a valuation $\nu$ is defined as $\alpha(\nu)=\sup _{\psi \in(x, y)} \frac{\nu(\psi)}{m_{O}(\psi)}$. Then it holds $\alpha\left(\nu_{C} \wedge \nu_{D}\right)=\frac{1}{d_{\mathcal{C}}(C, D)}$ (see Lemma 3.56 of [4]). Observe that in our context the definition of skewness of the valuation attached to a pair of branches can be translated to

$$
\alpha\left(\nu_{C} \wedge \nu_{D}\right)=\sup _{\psi \in(x, y) \text { irreducible }}\left(\min \left\{\frac{1}{d_{\mathcal{C}}(C,(\psi=0))}, \frac{1}{d_{\mathcal{C}}(D,(\psi=0))}\right\}\right),
$$

and hence this relation can be read as a sort of ultrametric inequality.

The aim of this paper is to obtain a deeper and geometrical insight of this distance $d_{\mathcal{C}}$. Namely, in Section 1 we give some preliminaries and recall some facts about the theory of infinitely near points, specially how they are combinatorially represented by Enriques diagrams and their use to classify singularities from the topological (or equisingular) point of view. In Section 2 we give tools to compute the distance between any two branches in a geometric way, and we state the main result of this section, which asserts that there are branches $D$ at any prescribed distance $d \in[0,1] \cap \mathbb{Q}$ of a given branch $C$. We also establish the relationship between the equisingularity classes of $C$ and $D$ and the rational number $d$. Finally in Section 3 we use the results developed in the preceding section to determine the relative position of three branches, classifying if the triangle (formed by the three branches) is isosceles or equilateral by a novel and straightforward method, just by inspection of their Enriques diagram and without further calculations.

\section{Preliminaries}

Here we review some basic notions and facts about singularity theory, specially those concerned with the topological equivalence of singularities of plane curves or equisingularity. The reader is referred to [1] chapter 3 for their proofs and an extensive exposition.

The distance $d_{\mathcal{C}}$ defined in equation (1) on the space of plane branches $\mathcal{C}$ is ultrametric, which means that for any three branches $C_{1}, C_{2}, C_{3} \in \mathcal{C}$ holds the ultrametric inequality

$$
d_{\mathcal{C}}\left(C_{1}, C_{2}\right) \leqslant \max \left\{d_{\mathcal{C}}\left(C_{1}, C_{3}\right), d_{\mathcal{C}}\left(C_{3}, C_{2}\right)\right\}
$$


instead of the usual (and weaker) triangular inequality $d_{\mathcal{C}}\left(C_{1}, C_{2}\right) \leqslant d_{\mathcal{C}}\left(C_{1}, C_{3}\right)+$ $d_{\mathcal{C}}\left(C_{3}, C_{2}\right)$ (see [5]).

Among the various consequences of this inequality, we will use the following one: if $d_{\mathcal{C}}\left(C_{1}, C_{2}\right) \neq d_{\mathcal{C}}\left(C_{1}, C_{3}\right)$, then $d_{\mathcal{C}}\left(C_{2}, C_{3}\right)=\max \left\{d_{\mathcal{C}}\left(C_{1}, C_{2}\right), d_{\mathcal{C}}\left(C_{1}, C_{3}\right)\right\}$ (which means that in a ultrametric space there are only equilateral and isosceles triangles).

Now let us switch to some theory of singularities of plane curves.

Let $\mathcal{N}_{O}$ denote the set of infinitely near points to $O$, which is constructed by successive blowing-ups. More precisely, if $\pi: \tilde{S} \rightarrow S$ denotes the blowing-up of $O$, the points in the exceptional divisor $E_{O}=\pi^{-1}(O)$ are the points in the first infinitesimal neighbourhood of $O$, and for every $i>1$ we define the points in the $i$-th infinitesimal neighbourhood of $O$ to be the points in the first neighbourhood of some point in the $(i-1)$-th neighbourhood of $O$. Now $\mathcal{N}_{O}$ is just the union of all the infinitesimal neighbourhoods of $O$. This set is equipped with a natural order: $p \leqslant q$ if and only if $q \in \mathcal{N}_{p}$ (read " $p$ precedes $q$ ").

For any $p \in \mathcal{N}_{O}$, let $\pi_{p}: S_{p} \rightarrow S$ be the minimal composition of blowing-ups such that $p$ appears as a proper point in a surface $S_{p}$. The germ at $p$ of the exceptional divisor $\pi_{p}^{-1}(O)$ consists of either one smooth curve or two smooth non-tangent branches. In the first case we say $p$ is a free point, and in the second case we call $p$ a satellite point. We say that a satellite point $q$ is satellite of $p$ (or $p$-satellite) if and only if $p$ is the maximal free point preceding $q$.

If $\xi$ is any germ of curve at $O$ defined by an equation $f=0$, we denote by $\bar{\xi}_{p}$ its total transform at $p$, which is defined as the germ of curve (at $p$ ) given by the equation $\pi_{p}^{*}(f)=f \circ \pi_{p}$. By subtracting from $\bar{\xi}_{p}$ the components contained in the exceptional divisor we obtain $\tilde{\xi}_{p}$, the strict transform of $\xi$ at $p$. The multiplicity of $\xi$ at the point $O$ is defined as the order of vanishing of $f$. The multiplicity of $\xi$ at a point $p \in \mathcal{N}_{O}$ is defined as $m_{p}(\xi)=m_{p}\left(\tilde{\xi}_{p}\right)$, the multiplicity of the corresponding strict transform. We also define the normalized multiplicity of $\xi$ at $p$ as the quotient $n_{p}(\xi)=\frac{m_{p}(\xi)}{m_{O}(\xi)}$, and $b_{p}(\xi)=n_{q}(\xi)$, where $q$ is the point immediately before $p$ (by the order $\leqslant$ ). By convention, set $b_{O}(\xi)=1$ for any curve $\xi$. If $m_{p}(\xi)>0$ we say that $p$ belongs to or lies on $\xi$, and also that $\xi$ goes through $p$. Denote $\mathcal{N}_{O}(\xi)=\mathcal{N}(\xi)=\left\{p \in \mathcal{N}_{O} \mid m_{p}(\xi)>0\right\}$ which will be called the set of (infinitely near) points on $\xi$.

Let $p, q \in \mathcal{N}_{O}$. We say that $q$ is proximate to $p$, denoted $q \rightarrow p$, if and only if $q$ belongs (as an ordinary or infinitely near point) to the exceptional divisor $E_{p}$ obtained by blowing up $p$. Note that free points are proximate to just one point (the one which precedes it), while satellite points are proximate to two points.

A finite subset $K \subseteq \mathcal{N}_{O}$ is a cluster if any point preceding $p \in K$ also belongs to $K$. Any cluster can be represented by means of an Enriques diagram, which is a rooted tree whose vertices are identified with the points in $K$ (the root corresponds to the origin $O$ ) and there is an edge between $p$ and $q$ if and only if $p$ lies on the first neighbourhood of $q$ or vice-versa. Moreover, the edges are drawn according to the following rules:

- If $q$ is free, proximate to $p$, the edge joining $p$ and $q$ is curved and if $p \neq O$, it is tangent to the edge ending at $p$. 
- If $p$ and $q$ ( $q$ in the first neighbourhood of $p$ ) have been represented, the rest of points proximate to $p$ in successive neighbourhoods of $q$ are represented on a straight half-line starting at $q$ and orthogonal to the edge ending at $q$. In this paper (and for the sake of clarity of the results stated in Section 3) we follow the extra convention that the first of these lines after each free point will be represented downwards and the next ones will be represented alternatively rightwards and downwards.

A point $p \in \mathcal{N}(\xi)$ is a singular point of $\xi$ if and only if the total transform $\bar{\xi}_{p}$ has non-normal crossings. Observe that the singular points of $\xi$ are precisely the multiple points and the simple points at which the strict transform of $\xi$ is tangent to the exceptional divisor. The set $S(\xi)$ consisting of the singular points and the minimal nonsingular points (by the order $\leqslant$ ) of $\xi$ is a cluster of $\mathcal{N}(\xi)$, and an Enriques diagram of $S(\xi)$ (or any cluster of $\mathcal{N}(\xi)$ containing it) will be called an Enriques diagram of the curve $\xi$. Two curves $\xi$ and $\zeta$ are called equisingular if there exists a bijection $\phi: S(\xi) \rightarrow S(\zeta)$ such that both $\phi$ and its inverse preserve natural order and proximity of infinitely near points. The importance of Enriques diagram is that they characterize the equisingularity (or topological equivalence) classes of plane curves.

With these definitions, the intersection multiplicity of two curves $\xi$ and $\zeta$ can be computed by means of the

Theorem 1.1 (Noether's intersection formula).

$$
[\xi \cdot \zeta]_{O}=\sum_{p \in \mathcal{N}(\xi) \cap \mathcal{N}(\zeta)} m_{p}(\xi) m_{p}(\zeta)
$$

in the sense that one side is finite if and only if the other side is, and in this case they are equal.

Recall that the multiplicities $m_{p}(\xi)$ of a curve $\xi$ are subjected to the

Proposition 1.2 (Proximity equalities).

$$
m_{p}(\xi)=\sum_{q \rightarrow p} m_{q}(\xi) .
$$

Now consider the case of an irreducible curve $C$. The set $\mathcal{N}(C)$ is totally ordered by $\leqslant$, and we also consider the set $\mathcal{F}(C)=\mathcal{F}_{O}(C)=\{O=$ $\left.p_{0}(C), p_{1}(C), p_{2}(C), \ldots\right\} \subseteq \mathcal{N}(C)$ of free points of $C$, which is also totally ordered by $\leqslant$. In this case denote $n_{k}(C)=n_{p_{k}(C)}(C)$ and $b_{k}(C)=b_{p_{k}(C)}(C)$.

The following property can be easily checked:

Lemma 1.3. $p_{k+1}(C)$ is proximate to $p_{k}(C)$ if and only if $b_{k}(C)=n_{k}(C)$.

\section{On the distance between branches}

In this section we develop some results concerning the computation of the distance between two branches $C$ and $D$ in $\mathcal{C}$. Then, we obtain the main re-

sult, which asserts that there are branches $D \in \mathcal{C}$ at any prescribed distance 
$d \in[0,1] \cap \mathbb{Q}$ of a given branch $C$. We show moreover that the distance $d$ determines to some extent the equisingularity class of $D$ in terms of the equisingularity class of $C$.

As it was said at the beginning, the geometric way we use to compute the distance between two branches $C$ and $D$ follows from Noether's intersection formula (Theorem 1.1), and it is given by

$$
\frac{1}{d_{\mathcal{C}}(C, D)}=\sum_{p \in \mathcal{N}(C) \cap \mathcal{N}(D)} n_{p}(C) n_{p}(D) .
$$

Our first aim is to obtain a formula involving only the common free points of $C$ and $D$, and to achieve it we need to develop some preliminary results.

We begin by considering sequences of consecutive satellite points on a branch $C$. Let $p \in \mathcal{F}(C)$ be a free point different from $O$ and proximate to $p^{\prime} \in \mathcal{N}(C)$ (note that $p^{\prime}$ need not be free), and let also $m_{0}=m_{p^{\prime}}(C)$ and $m_{1}=m_{p}(C)$ be the multiplicities of $C$ at these points. Let $a_{1}, \ldots, a_{r}$ and $m_{2}, \ldots, m_{r}$ be defined by Euclid's division algorithm as $m_{0}=a_{1} m_{1}+m_{2}, m_{1}=a_{2} m_{2}+$ $m_{3}, \ldots, m_{r-1}=a_{r} m_{r}$. By Theorems 3.5.8 and 5.5.1 of [1], the sequence of points on $C$ after $p^{\prime}$ begins with

$$
p_{1,1}=p<\ldots<p_{1, a_{1}}<p_{2,1}<\ldots<p_{2, a_{2}}<\ldots<p_{r, 1}<\ldots<p_{r, a_{r}}
$$

where each point has multiplicity $m_{p_{i, j}}(C)=m_{i}$, every point but $p=p_{1,1}$ is satellite, and the point on $C$ after $p_{r, a_{r}}$ is free. We denote this set by $\mathcal{N}^{p}(C)$. Moreover, the points $p_{i, 1}, \ldots, p_{i, a_{i}}, p_{i+1,1}$ are proximate to $p_{i-1, a_{i-1}}$, setting $p^{\prime}=p_{0,0}, a_{0}=0$, and $p_{r, a_{r}}=p_{r+1,1}$ by convention. Notice that the $p$-satellite points themselves and the multiplicities of $C$ at them are determined just by the multiplicities of $C$ at $p^{\prime}$ and $p$, and in fact by the quotient $\frac{m_{p^{\prime}}(C)}{m_{p}(C)}=\frac{b_{p}(C)}{n_{p}(C)}$. We will refer to this fact as the distribution of satellite points.

Remark 2.1. Keep the above notations. The distribution of satellite points can be alternatively obtained from the expansion in continued fraction of the quotient $\frac{m_{p^{\prime}}(C)}{m_{p}(C)}=\frac{b_{p}(C)}{n_{p}(C)}$. In fact,

$$
\frac{m_{p^{\prime}}(C)}{m_{p}(C)}=\left[a_{1}, a_{2}, \ldots, a_{r}\right]=a_{1}+\frac{1}{a_{2}+\frac{1}{\ddots \cdot+\frac{1}{a_{r}}}} .
$$

The distribution of the p-satellite points is then shown in the Enriques diagram of $C$ by $r$ sides of stairs with lengths $a_{1}$ (counting $p$ ), $a_{2}, \ldots, a_{r}$ after $p^{\prime}$ (see Figure 1).

So it seems feasible to simplify expression (2), replacing the contribution of the set of $p$-satellite points of $C$ by some expression depending only of $b_{p}(C)$ and $n_{p}(C)$. The main tool to do this is next 


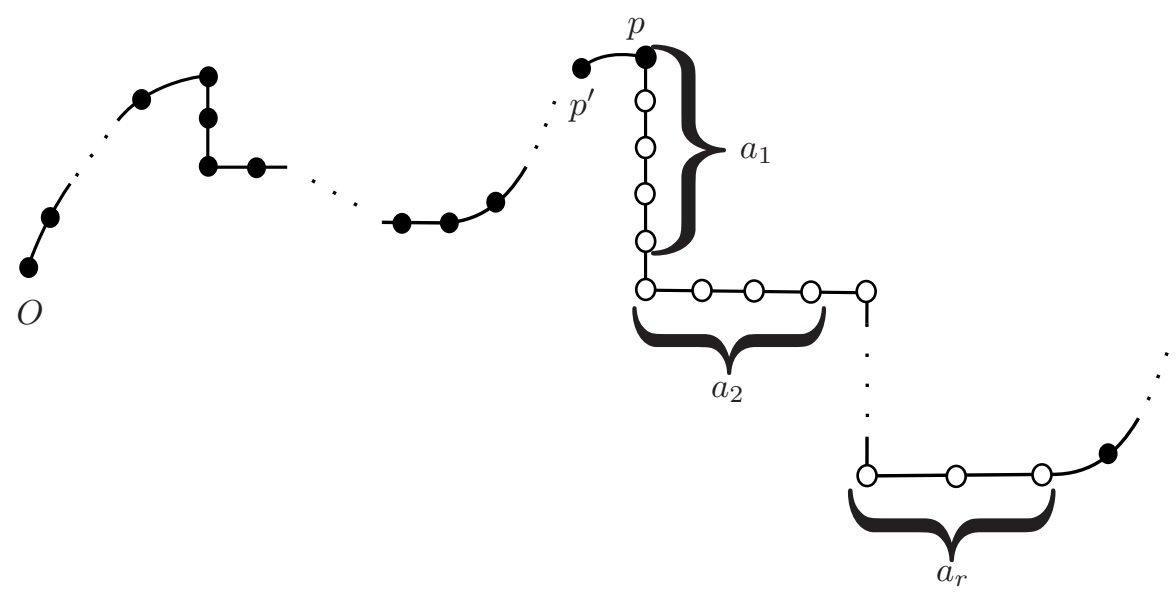

Figure 1: The distribution of satellite points in the Enriques diagram. In this picture, $p$-satellite points are represented by white-filled circles.

Lemma 2.2. Let $0<m_{1} \leqslant m_{0}$ be two natural numbers. Let $m_{0}=a_{1} m_{1}+$ $m_{2}, m_{1}=a_{2} m_{2}+m_{3}, \ldots, m_{r-1}=a_{r} m_{r}$ be the divisions performed by Euclid's algorithm. Then $m_{1} m_{0}=a_{1} m_{1}^{2}+a_{2} m_{2}^{2}+\cdots+a_{r} m_{r}^{2}$.

Proof. We argue by induction on $r$. If $r=1$, then $m_{0}=a_{1} m_{1}$, so $m_{0} m_{1}=a_{1} m_{1}^{2}$. In the general case, we apply the induction hypothesis on $m_{1}$ and $m_{2}: m_{1} m_{2}=$ $a_{2} m_{2}^{2}+\cdots+a_{r} m_{r}^{2}$. So $m_{0} m_{1}=\left(a_{1} m_{1}+m_{2}\right) m_{1}=a_{1} m_{1}^{2}+a_{2} m_{2}^{2}+\cdots+a_{r} m_{r}^{2}$.

As an immediate consequence we obtain

Corollary 2.3. If $C \in \mathcal{C}$ and $p \in \mathcal{N}(C)$ is a free point different from $O$ then

$$
\sum_{q \in \mathcal{N}^{p}(C)} n_{q}(C)^{2}=n_{p}(C) b_{p}(C) .
$$

Proof. Let $p^{\prime}$ be the immediate predecessor of $p$ (so that $b_{p}(C)=m_{p^{\prime}}(C) / m_{O}(C)$ ) and define $m_{0}=m_{p^{\prime}}(C)$ and $m_{1}=m_{p}(C)$. Then, using the preceding notations and Lemma 2.2 we obtain, as desired,

$$
\begin{aligned}
\sum_{q \in \mathcal{N}^{p}(C)} n_{q}(C)^{2} & =\frac{1}{m_{O}(C)^{2}} \sum_{i=1}^{r} \sum_{j=1}^{a_{i}} m_{p_{i, j}}(C)^{2}=\frac{1}{m_{O}(C)^{2}} \sum_{i=1}^{r} a_{i} m_{i}^{2} \\
& =\frac{1}{m_{O}(C)^{2}} m_{0} m_{1}=\frac{1}{m_{O}(C)^{2}} m_{p^{\prime}}(C) m_{p}(C)=b_{p}(C) n_{p}(C) .
\end{aligned}
$$

Lemma 2.4. Let $C, D$ be two branches, and suppose that $p$ is the last free point in $\mathcal{N}(C) \cap \mathcal{N}(D)$. Then $n_{q}(C)=n_{q}(D)$ for any $q<p$, or, in other words, $b_{q}(C)=b_{q}(D)$ for any $q \leqslant p$. 
Proof. Suppose that $q<p$ is the first common free point for which $n_{q}(C) \neq$ $n_{q}(D)$. In this case, $n_{q^{\prime}}(C)=n_{q^{\prime}}(D)$ for all free or satellite points $q^{\prime}$ with $O \leqslant q^{\prime}<q$, since, by the distribution of satellite points, the multiplicities at the satellite points are determined by the multiplicities at the preceding free point and the point to which it is proximate. Therefore, $b_{q}(C)=b_{q}(D)$, so $\frac{n_{q}(C)}{b_{q}(C)} \neq \frac{n_{q}(D)}{b_{q}(D)}$. Hence, invoking the distribution of satellite points again, the branches $C$ and $D$ split either at $q$ or at a $q$-satellite point. This is a contradiction with the definition of $p$.

Now we are ready to prove the next proposition, which plays a key role in our main result.

Proposition 2.5. Let $C, D$ be two branches. Then

$$
\frac{1}{d_{\mathcal{C}}(C, D)}=\sum b_{k}(C) \min \left\{n_{k}(C), n_{k}(D)\right\}
$$

where the summation runs over all points $p_{k} \in \mathcal{F}(C) \cap \mathcal{F}(D)$.

Remark 2.6. Notice that, according to Lemma 2.4, for every point $p_{k} \in \mathcal{F}(C) \cap$ $\mathcal{F}(D)$ we have the equality $b_{k}(C)=b_{k}(D)$, and for every such point but perhaps the last one we also have $n_{k}(C)=n_{k}(D)$. Thus, the statement of Proposition 2.5 may be simplified as

$$
\frac{1}{d_{\mathcal{C}}(C, D)}=\sum_{k=0}^{N-1} b_{k}(C) n_{k}(C)+b_{N}(C) \min \left\{n_{N}(C), n_{N}(D)\right\}
$$

where $\mathcal{F}(C) \cap \mathcal{F}(D)=\left\{p_{0}=O, p_{1}, \ldots, p_{N}\right\}$.

Proof of Proposition 2.5. Let $p_{0}=O, p_{1}, \ldots, p_{N}$ be the common free points of $C$ and $D$. Then, by 2.6 we have $n_{k}(C)=n_{k}(D)$ for $k<N$, and interchanging $C$ and $D$ if necessary, we may assume that $n_{N}(C) \leqslant n_{N}(D)$. With this assumptions, we have to prove that

$$
\frac{1}{d_{\mathcal{C}}(C, D)}=\sum_{k=0}^{N} b_{k}(C) n_{k}(C)
$$

First of all, using Lemma 2.4 the summands in expression (2) can be grouped as

$$
\sum_{q \in \mathcal{N}^{p_{0}}(C)} n_{q}(C)^{2}+\cdots+\sum_{q \in \mathcal{N}^{p_{N}-1}(C)} n_{q}(C)^{2}+\sum_{q \in \mathcal{N}^{p_{N}}(C) \cap \mathcal{N}^{p_{N}}(D)} n_{q}(C) n_{q}(D) .
$$

By Corollary 2.3, for $k<N$ the partial sum $\sum_{q \in \mathcal{N}^{p} p_{k}(C)} n_{q}(C)^{2}$ equals $b_{k}(C) n_{k}(C)$, so we have proved that

$$
\frac{1}{d_{\mathcal{C}}(C, D)}=\sum_{k=0}^{N-1} b_{k}(C) n_{k}(C)+\sum_{q \in \mathcal{N}^{p_{N}}(C) \cap \mathcal{N}^{p_{N}}(D)} n_{q}(C) n_{q}(D)
$$


and it only remains to show that the last summand equals $b_{N}(C) n_{N}(C)$.

If $n_{N}(C)=n_{N}(D)$, then $\mathcal{N}^{p_{N}}(C)=\mathcal{N}^{p_{N}}(D)$ and $n_{q}(C)=n_{q}(D)$ for all $q \in \mathcal{N}(C) \cap \mathcal{N}(D)$, and the desired result follows applying Corollary 2.3 as for the other sums. If $n_{N}(C)<n_{N}(D)$, consider a branch $E$ through $p_{N}$ whose point in its first neighbourhood is free and does not belong to $D$ (nor to $C$, since $n_{N}(C)<n_{N}(D) \leqslant b_{N}(D)=b_{N}(C)$ implies that the immediate successor of $p$ on $C$ is satellite). Applying Lemma 2.4 and what we have already proved, and taking into account that $\mathcal{N}^{p_{N}}(C) \cap \mathcal{N}^{p_{N}}(E)=\mathcal{N}^{p_{N}}(D) \cap \mathcal{N}^{p_{N}}(E)=\left\{p_{N}\right\}$ and that $n_{N}(E)=b_{N}(C)=b_{N}(D)$, we obtain

$$
\begin{aligned}
d_{\mathcal{C}}(C, E)^{-1}=\sum_{k=0}^{N-1} b_{k}(C) & n_{k}(C)+b_{N}(C) n_{N}(C)=\sum_{k=0}^{N} b_{k}(C) n_{k}(C)< \\
& <\sum_{k=0}^{N-1} b_{k}(C) n_{k}(C)+b_{N}(C) n_{N}(D)=d_{\mathcal{C}}(D, E)^{-1} .
\end{aligned}
$$

Hence, $d_{\mathcal{C}}(C, E)>d_{\mathcal{C}}(D, E)$; since $d_{\mathcal{C}}$ is an ultrametric distance, we derive $d_{\mathcal{C}}(C, D)=d_{\mathcal{C}}(C, E)$, and we are done.

Now we can prove the main result of this section.

Theorem 2.7. Let $C$ be any branch and take $d \in \mathbb{Q} \cap[0,1]$. Then there exists a branch $D \in \mathcal{C}$ for which $d_{\mathcal{C}}(C, D)=d$.

Furthermore, if $d>0$, any such $D$ shares with $C$ exactly the first $N+1$ points in $\mathcal{F}(C)$, where

$$
\sum_{k=0}^{N-1} b_{k}(C) n_{k}(C)<d^{-1} \leqslant \sum_{k=0}^{N} b_{k}(C) n_{k}(C) .
$$

Proof. If $d=0$ the result is obvious, by taking $D=C$, so we can assume $d>0$. The series $\sum_{k \geqslant 0} b_{k}(C) n_{k}(C)$ does not converge because $d_{\mathcal{C}}(C, C)=0$, and its partial sums form a strictly increasing sequence (since every summand is positive). So there exists $N \in \mathbb{N}$ for which

$$
\sum_{k=0}^{N-1} b_{k}(C) n_{k}(C)<\frac{1}{d} \leqslant \sum_{k=0}^{N} b_{k}(C) n_{k}(C) .
$$

If we have equality, by Proposition 2.5 and Lemma 2.4 it is enough to take any branch $D$ going through $p_{N}$ with normalized multiplicity $n_{N}(D)=n_{N}(C)$, but not through $p_{N+1}$. Notice that this kind of curves exist in virtue of Theorem 4.2.2 of $[1]$.

Suppose now that the second inequality is strict, and define $\alpha \in \mathbb{Q}$ as

$$
\alpha=\frac{d^{-1}-\sum_{k=0}^{N-1} b_{k}(C) n_{k}(C)}{b_{N}(C)} .
$$


It is obvious that $\alpha<n_{N}(C)$ (because of the second inequality in (3)), and again by Proposition 2.5 and Lemma 2.4, it is enough to take any branch $D$ going through $p_{N}$ with normalized multiplicity $n_{N}(D)=\alpha<n_{N}(C)$ (without any assumption on the next free point on $D$ ), whose existence is again guaranteed by Theorem 4.2 .2 of $[1]$.

On the other hand, let $D$ be a branch such that $d_{\mathcal{C}}(C, D)=d>0$. Then, by Remark 2.6 and by the definition of $N, C$ and $D$ must share the first $N+1$ points of $\mathcal{F}(C)$.

Corollary 2.8. Let $C \in \mathcal{C}$ and $d \in \mathbb{Q} \cap[0,1]$ and compute $N$ and $\alpha$ such that

$$
\sum_{k=0}^{N-1} b_{k}(C) n_{k}(C)<d^{-1} \leqslant \sum_{k=0}^{N} b_{k}(C) n_{k}(C), \quad \alpha=\frac{d^{-1}-\sum_{k=0}^{N-1} b_{k}(C) n_{k}(C)}{b_{N}(C)} .
$$

If $D \in \mathcal{C}$ satisfies $d_{\mathcal{C}}(C, D)=d$ and $D$ has only p-satellite points for $p \in \mathcal{F}(C)$, then the equisingularity class of $C+D$ is completely determined by $C$ and $d$ if and only if either $\alpha>0$, or $p_{N+1}(C)$ is proximate to $p_{N}(C)$.

Proof. First, let us assume that $\alpha=0$. By the previous Theorem, $C$ and $D$ share exactly $N+1$ free points, and, since $\alpha=0$,

$d_{C}(C, D)^{-1}=\sum_{k=0}^{N} b_{k}(C) n_{k}(C)=\sum_{k=0}^{N-1} b_{k}(C) n_{k}(C)+b_{N}(C) \min \left\{n_{N}(C), n_{N}(D)\right\}$

in virtue of Remark 2.6.

Therefore, $n_{N}(C) \leqslant n_{N}(D)$. If $p_{N+1}(C)$ is proximate to $p_{N}(C)$, then $n_{N}(C)=b_{N}(C)=b_{N}(D) \geqslant n_{N}(D)$ (by Lemma 2.4). Then, the point in the first neighbourhood of $p_{N}(D)$ on $D$ is free, and nonsingular (since $D$ has no $p$-satellite points for $p \notin \mathcal{F}(C))$. The Enriques diagram of $C+D$ is thus determined, therefore also its equisingularity class.

On the other hand, if $p_{N+1}(C)$ is not proximate to $p_{N}(C), n_{N}(C)<b_{N}(C)=$ $b_{N}(D)$. Therefore, any curve $D$ going through $p_{N}(C)$ with normalized multiplicity $n_{N}(D) \in\left(n_{N}(C), b_{N}(D)\right] \cap \mathbb{Q}$ satisfies $d_{\mathcal{C}}(C, D)=d$. But in virtue of Remark 2.1, if two curves have different values of $n_{N}(D)$, they belong to different equisingularity classes.

Now assume that $\alpha>0$. In this case, by Theorem 2.7, $C$ and $D$ share exactly $N+1$ free points. By Remark 2.6,

$$
d_{C}(C, D)^{-1}=\sum_{k=0}^{N-1} b_{k}(C) n_{k}(C)+b_{N}(C) \min \left\{n_{N}(C), n_{N}(D)\right\},
$$

so $\alpha=\min \left\{n_{N}(C), n_{N}(D)\right\}$. On the other hand, by the definition of $N, \alpha<$ $n_{N}(C)$. Therefore, $\alpha=n_{N}(D)<n_{N}(C) \leqslant b_{N}(C)=b_{N}(D)$. By Remark 2.1, the $p_{N}(D)$-satellite points of $D$ are determined by $n_{N}(D)=\alpha$ and $b_{N}(D)=$ $b_{N}(C)$. Since $D$ has no $p$-satellite points for $p \notin \mathcal{F}(C)$, this determines its Enriques diagram. 
Example 2.9. Let us compute the distance between the branches $C: y^{11}-x^{29}=$ 0 and $D: y^{12}-x^{31}=0$ (see Figure 2). When the two branches have the same normalized multiplicity at a point $p_{i}$, we refer to it as $n_{i}=n_{i}(C)=n_{i}(D)$ (the same for $\left.b_{i}=b_{i}(C)=b_{i}(D)\right)$. Then, the branches share three free points and $n_{0}=n_{1}=b_{0}=b_{1}=b_{2}=1, n_{2}(C)=7 / 11$ and $n_{2}(D)=7 / 12$. Therefore, their distance is $d_{\mathcal{C}}(C, D)=12 / 31$. Notice that in this case $d_{\mathcal{C}}(C, D)$ is exactly the inverse of the minimum of the first characteristic exponents of $C$ and of $D$. This occurs because $C$ and $D$ split up at the first stairs of satellite points. Otherwise the computation of $d_{\mathcal{C}}(C, D)$ becomes more involved.

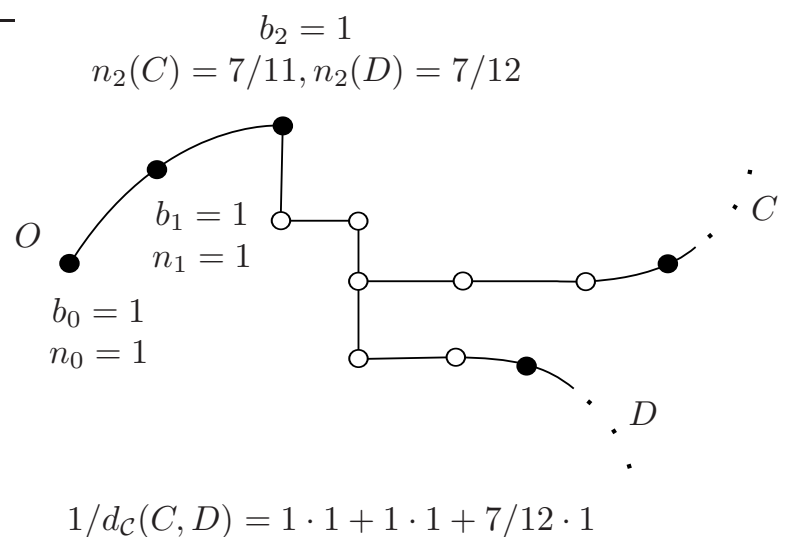

Figure 2: Computation of the distance between the two branches $C$ and $D$ appearing in Example 2.9, together with the Enriques diagram of $C+D$. Free points are drawn in black-filled circles, whereas satellite points are drawn in white-filled circles.

\section{Triangles in the ultrametric space of plane branches}

In this section the relative position of three branches is compared by using the ultrametric inequality. In spaces which are equipped with an ultrametric distance, the triangles can only be isosceles or equilateral. Thus, given three branches there are only two possibilities: either they form an equilateral triangle, or two of them are nearer one from each other and equidistant from the third one. Next we state the two results that solve this question:

Theorem 3.1. Let $C_{1}, C_{2}, C_{3}$ be three different branches. Assume that there is a free point shared by $C_{1}$ and $C_{2}$ but not by $C_{3}$. Then, the three branches form an isosceles triangle, where $C_{1} C_{2}$ is the shortest side.

Theorem 3.2. Let $C_{1}, C_{2}, C_{3}$ be three branches sharing the same free points, and let $p_{N}$ be the last common free point. Assume that $n_{N}\left(C_{1}\right) \geqslant n_{N}\left(C_{2}\right) \geqslant$ 
$n_{N}\left(C_{3}\right)$. Then, the three curves form an equilateral triangle if and only if $n_{N}\left(C_{2}\right)=n_{N}\left(C_{3}\right)$. Otherwise they form an isosceles triangle with $C_{1} C_{2}$ as the shortest side.

Notice that the hypothesis of these two theorems cover every possibility, so they completely solve the classification problem of triangles. Let us discuss first the result of Theorem 3.2.

Proof of Theorem 3.2. First of all, denote $b_{k}=b_{k}\left(C_{i}\right)$ for $k \leqslant N$ and $n_{k}=$ $n_{k}\left(C_{i}\right)$ for $k<N$ (which do not depend on $i$ by Lemma 2.4). In virtue of Proposition 2.5 and the hypotheses on the $n_{N}\left(C_{i}\right)$, the distances between the curves may be written

$$
d_{\mathcal{C}}\left(C_{1}, C_{2}\right)^{-1}=\sum_{k=0}^{N-1} b_{k} n_{k}+b_{N} n_{N}\left(C_{2}\right)
$$

and

$$
d_{\mathcal{C}}\left(C_{1}, C_{3}\right)^{-1}=d_{\mathcal{C}}\left(C_{2}, C_{3}\right)^{-1}=\sum_{k=0}^{N-1} b_{k} n_{k}+b_{N} n_{N}\left(C_{3}\right) .
$$

The result follows by considering the cases $n_{N}\left(C_{2}\right)>n_{N}\left(C_{3}\right)$ and $n_{N}\left(C_{2}\right)=$ $n_{N}\left(C_{3}\right)$.

Figure 3 illustrates several cases of triangles occurring when the three branches share the same free points.

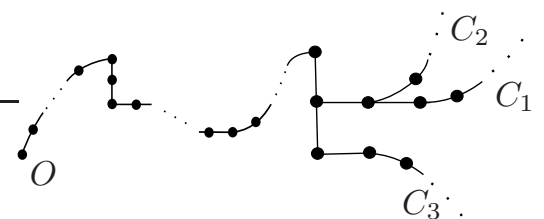

$n_{N}\left(C_{1}\right)>n_{N}\left(C_{2}\right)>n_{N}\left(C_{3}\right)$ :

An isosceles triangle.

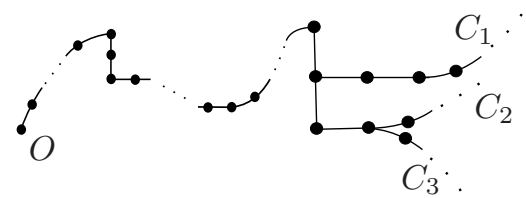

$$
n_{N}\left(C_{1}\right)>n_{N}\left(C_{2}\right)=n_{N}\left(C_{3}\right):
$$

An equilateral triangle.

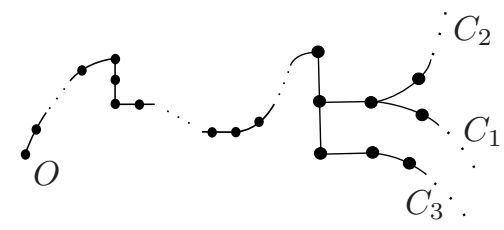

$n_{N}\left(C_{1}\right)=n_{N}\left(C_{2}\right)>n_{N}\left(C_{3}\right)$ :

An isosceles triangle.

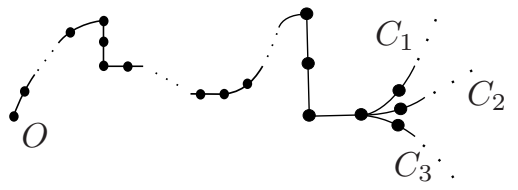

$n_{N}\left(C_{1}\right)=n_{N}\left(C_{2}\right)=n_{N}\left(C_{3}\right)$ :

An equilateral triangle.

Figure 3: Different kinds of triangles formed by curves sharing the same free points. 
Remark 3.3. Notice that, given $C_{2}$ and $C_{3}$ with $n_{N}\left(C_{2}\right) \geqslant n_{N}\left(C_{3}\right)$, the triangle formed by $C_{2}, C_{3}$ and any other curve $C_{1}$ is either isosceles or equilateral regardless $C_{1}$ as long as $C_{1}, C_{2}$ and $C_{3}$ share the same free points and $n_{N}\left(C_{1}\right)$ is in the interval $\left[n_{N}\left(C_{2}\right), b_{N}\left(C_{2}\right)\right]$. For example, in Figure 4 triangles $C_{1} C_{2} C_{3}$ and $C_{1}^{\prime} C_{2} C_{3}$ are equilateral.

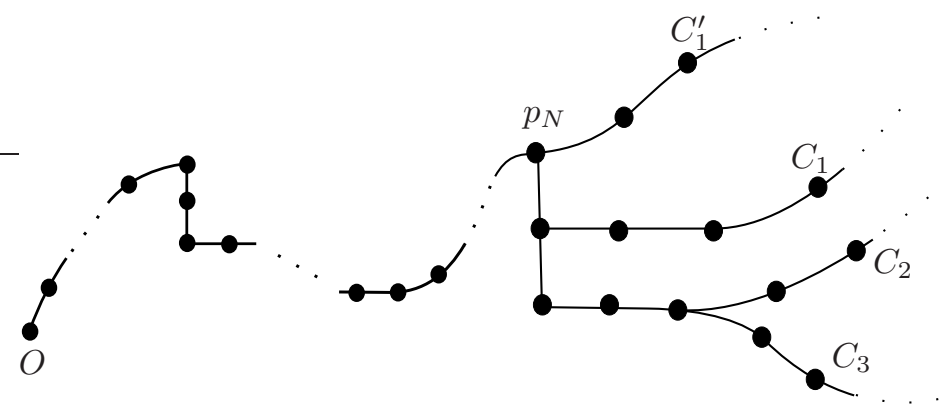

Figure 4: $C_{1} C_{2} C_{3}$ and $C_{1}^{\prime} C_{2} C_{3}$ are equilateral triangles.

Proof of Theorem 3.1. Let $p_{N}$ be the last free point shared by the three branches. Let $p_{N+1}$ be the first free point after $p_{N}$ lying on both $C_{1}$ and $C_{2}$. Notice that by Lemma $2.4 n_{N}\left(C_{1}\right)=n_{N}\left(C_{2}\right)$ since there is a free point shared by $C_{1}$ and $C_{2}$ after $p_{N}$.

First, assume that $n_{N}\left(C_{3}\right) \leqslant n_{N}\left(C_{1}\right)=n_{N}\left(C_{2}\right)$. In this case, and in virtue of Proposition 2.5, we obtain the desired result

$$
\frac{1}{d_{\mathcal{C}}\left(C_{1}, C_{2}\right)}>\sum_{k=0}^{N} b_{k} n_{k}\left(C_{1}\right) \geqslant \sum_{k=0}^{N} b_{k} n_{k}\left(C_{3}\right)=\frac{1}{d_{\mathcal{C}}\left(C_{1}, C_{3}\right)}=\frac{1}{d_{\mathcal{C}}\left(C_{2}, C_{3}\right)} .
$$

Notice that the first inequality is strict because $C_{1}$ and $C_{2}$ share more free points.

Now assume $n_{N}\left(C_{3}\right)>n_{N}\left(C_{1}\right)=n_{N}\left(C_{2}\right)$ and take an auxiliary branch $D$ forming an equilateral triangle with $C_{1}$ and $C_{3}$ and an isosceles one with $C_{1}$ and $C_{2}$ : let $D$ be a branch going through $p_{N}$ with $n_{N}(D)=n_{N}\left(C_{1}\right)$ and not going through $p_{N+1}$ (which exists in virtue of Theorem 4.2.2 of [1]). Then $C_{3}, D$ and $C_{1}$ share the same free points, and we are under the hypothesis of Theorem 3.2. As $n_{N}\left(C_{3}\right)>n_{N}(D)=n_{N}\left(C_{1}\right)$ by assumption, the three curves form an equilateral triangle. Therefore:

$$
d_{\mathcal{C}}\left(C_{1}, D\right)=d_{\mathcal{C}}\left(C_{1}, C_{3}\right)=d_{\mathcal{C}}\left(C_{3}, D\right) .
$$

On the other hand, in virtue of Proposition 2.5,

$$
\frac{1}{d_{\mathcal{C}}\left(C_{1}, D\right)}=\sum_{k=0}^{N} b_{k} n_{k}\left(C_{1}\right)<\frac{1}{d_{\mathcal{C}}\left(C_{1}, C_{2}\right)} .
$$


Notice that the inequality is strict because $C_{1}$ and $C_{2}$ share more free points than the points $\left\{p_{0}, \ldots, p_{N}\right\}$ in the sum. Therefore, $d_{\mathcal{C}}\left(C_{1}, D\right)>d_{\mathcal{C}}\left(C_{1}, C_{2}\right)$. Combining this with (4), we obtain the statement of Theorem 3.1 also in this case.

To conclude the classification of triangles, we give a method for the comparison between distances of three branches just by observing the Enriques diagrams, and without making any further calculations.

We can distinguish easily free points from satellite ones in the Enriques diagram of any curve. Thus, from an Enriques diagram of $C_{1}+C_{2}+C_{3}$ it is also straightforward to find which free points are shared by two or three branches. Therefore, it is easy to decide whether to use Theorem 3.2 or Theorem 3.1 by direct observation of the diagram involved. In the case of this latter Theorem, the Enriques diagram also shows which branches share more free points. Thus, we can know which two branches form the shortest side of the triangle.

If we are dealing with a case where the three branches share the same free points, we only need to compare the normalized multiplicities of the branches at their last free shared point. Let $C_{1}, C_{2}$ be two branches splitting up at a satellite point $q$, and let $p=p_{N}$ be their last common free point. Denote by $q_{i}$ the point of $C_{i}$ in the first neighbourhood of $q$ and $n_{i}=n_{p}\left(C_{i}\right)=n_{N}\left(C_{i}\right)$. Let us draw the Enriques diagram of the curve $C_{1}+C_{2}$ (see Figure 5). The segment between $p$ and the satellite point in the first neighbourhood of $p$ should be vertical following the convention of Section 1 . Then, either $q_{1}$ is a free point, or $q_{1}$ is on the right of $q$, or $q_{1}$ is under $q$ (idem for $q_{2}$ ). Then

Proposition 3.4. Keeping notation as above:

1. If $q_{i}$ is on the right side of $q$, then $n_{N}\left(C_{i}\right)>n_{N}\left(C_{j}\right)$, with $j \in\{1,2\}, j \neq i$.

2. If $q_{i}$ is under $q$, then $n_{N}\left(C_{i}\right)<n_{N}\left(C_{j}\right)$, with $j \in\{1,2\}, j \neq i$.

3. If $q_{1}$ and $q_{2}$ are both free, then $n_{N}\left(C_{1}\right)=n_{N}\left(C_{2}\right)$.

Proof. First of all, notice that the different statements of the Proposition are compatible, namely it is impossible that $q_{1}$ and $q_{2}$ are both on the right of $q$, because in this case $q_{1}=q_{2}$ contradicting the definition of $q$ (idem if $q_{1}$ and $q_{2}$ are both under $q$ ). On the other side, if $q_{1}$ is on the right of $q$ and $q_{2}$ is under it, then first and second statements say that $n_{N}\left(C_{1}\right)>n_{N}\left(C_{2}\right)$.

Assume that we have the expansions in continued fractions

$\frac{b_{N}\left(C_{1}\right)}{n_{N}\left(C_{1}\right)}=\left[a_{1}, a_{2}, \ldots, a_{k}, a_{k+1}, \ldots, a_{r}\right], \frac{b_{N}\left(C_{2}\right)}{n_{N}\left(C_{2}\right)}=\left[a_{1}, a_{2}, \ldots, a_{k}, b_{k+1}, \ldots, b_{s}\right]$,

with $a_{r}, b_{s}>1$. Suppose that either $k=r$, or $k=s$, or $a_{k+1} \neq b_{k+1}$. Notice that the condition $n_{N}\left(C_{1}\right)>n_{N}\left(C_{2}\right)$ is equivalent to $\left[a_{1}, a_{2}, \ldots, a_{k}, a_{k+1}, \ldots, a_{r}\right]<$ $\left[a_{1}, a_{2}, \ldots, a_{k}, b_{k+1}, \ldots, b_{s}\right]$.

On the other side, the condition of $q_{1}$ being on the right side of $q$ is equivalent to either $k$ being even and $k=s<r$ (which happens if and only if $q$ is the last 


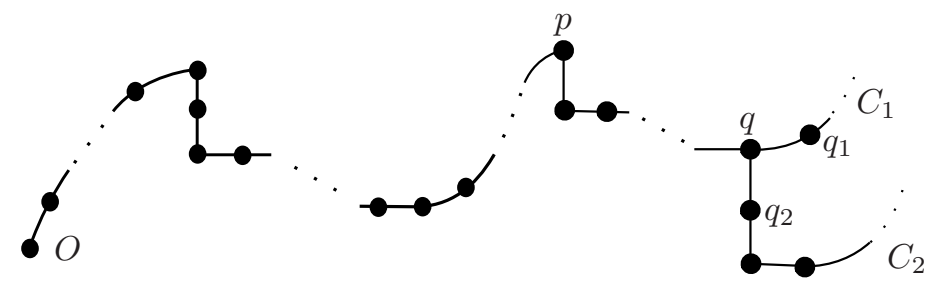

Example A: $q_{1}$ is free and $q_{2}$ is under $q$.

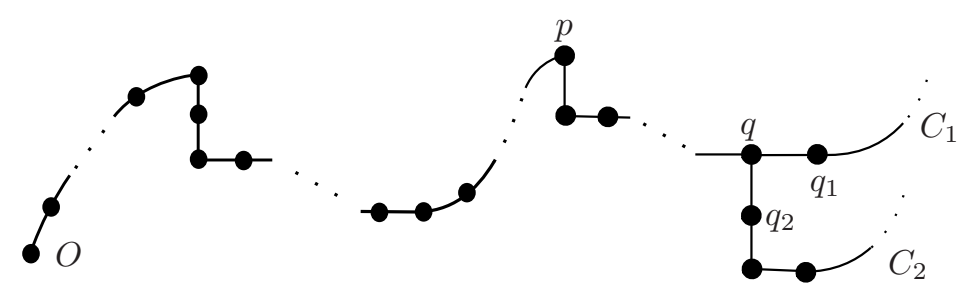

Example B: $q_{1}$ is on the right of $q$ and $q_{2}$ is under $q$.

Figure 5: Two examples of branches $C_{1}$ and $C_{2}$ splitting up at a satellite point $q$.

$p$-satellite point on $C_{2}$ ), or $k$ being even and $a_{k+1}<b_{k+1}$, or $k$ being odd and $a_{k+1}>b_{k+1}$.

Similarly, the condition of $q_{1}$ being under $q$ is equivalent to either $k$ being odd and $k=s<r$ (which again happens if and only if $q$ is the last $p$-satellite point on $C_{2}$ ), or $k$ being even and $a_{k+1}>b_{k+1}$, or $k$ being odd and $a_{k+1}<b_{k+1}$.

Lastly, the condition of $q_{1}$ and $q_{2}$ being free is equivalent to $r=s=k$.

The proof is completed in virtue of the forthcoming elementary Lemma 3.5 about comparison between continued fractions.

Lemma $3.5([3], \S 12)$. Consider the expansions in continued fractions

$$
x=\left[a_{1}, a_{2}, \ldots, a_{k}, a_{k+1}, \ldots, a_{r}\right], \quad y=\left[a_{1}, a_{2}, \ldots, a_{k}, b_{k+1}, \ldots, b_{s}\right],
$$

with $a_{r}>1$ and $b_{s}>1$.

- If $r>k, s>k$ and $a_{k+1}>b_{k+1}$, then $x>y$ if $k$ is even, and $x<y$ otherwise.

- If $r>k$ and $s=k$, then $x>y$ if $k$ is even, and $x<y$ otherwise.

- If $r=s=k$, then $x=y$. 

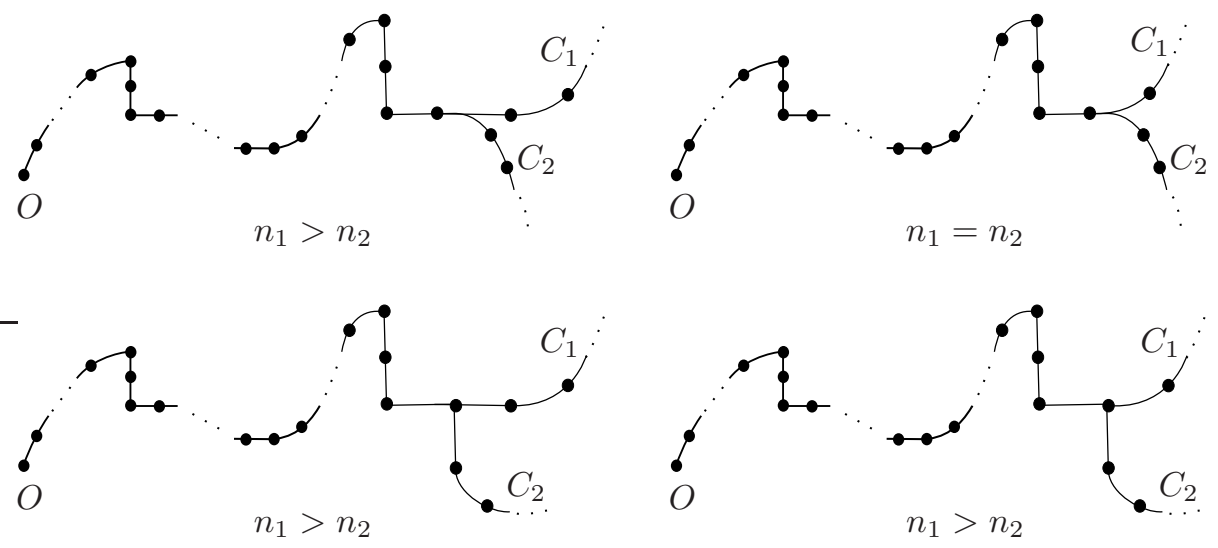

Figure 6: Illustration of the different ways of splitting up at a satellite point.

\section{References}

[1] E. Casas-Alvero, Singularities of Plane Curves, London Math. Soc. Lecture Note Ser., vol. 276, Cambridge University Press, 2000.

[2] E. Casas-Alvero, Local Geometry of Planar Analytic Morphisms. Asian J. Math. Volume 11, Number 3 (2007), pp. 373-426.

[3] O. Perron, Die Lehre von den Kettenbrüchen. Bd I. Elementare Kettenbrüche, B. G. Teubner Verlagsgesellschaft, Stuttgart, 1954.

[4] C. Favre and M. Jonsson, The Valuative Tree, Lecture Notes in Mathematics, vol. 1853, Springer-Verlag, Berlin, 2004.

[5] A Płoski. Remarque sur la multiplicité d'intersection des branches planes. Bulletin of the Polish Academy of Sciences Mathematics. Volume 33 (1985), pp. 601-605.

[6] M. Merle, Invariants polaires des courbes planes, Invent. Math. Volume 41 (1977), pp. 103-111.

[7] C.T.C. Wall, Singular points of plane curves. ondon Math. Soc. Student Texts, vol. 63, Cambridge University Press, 2004. 